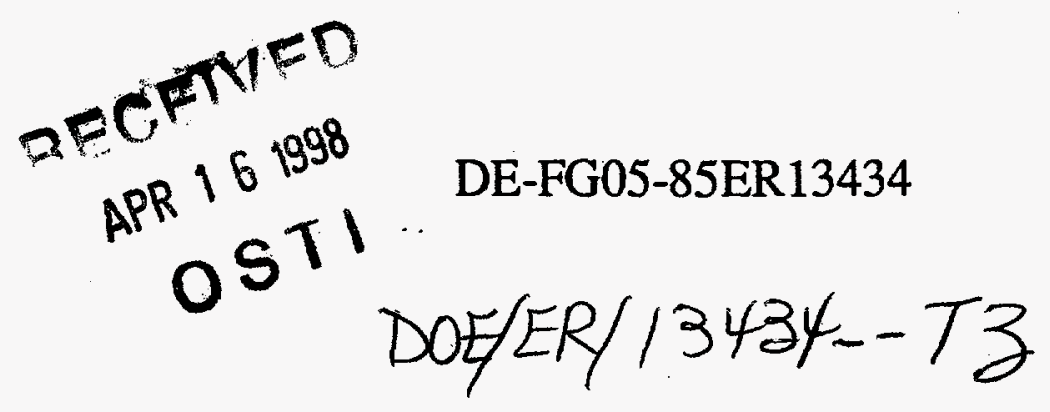

\title{
DEVELOPMENT OF LASER-ION BEAM PHOTODISSOCIATION METHODS
}

Progress Report

December 1991-November 1994

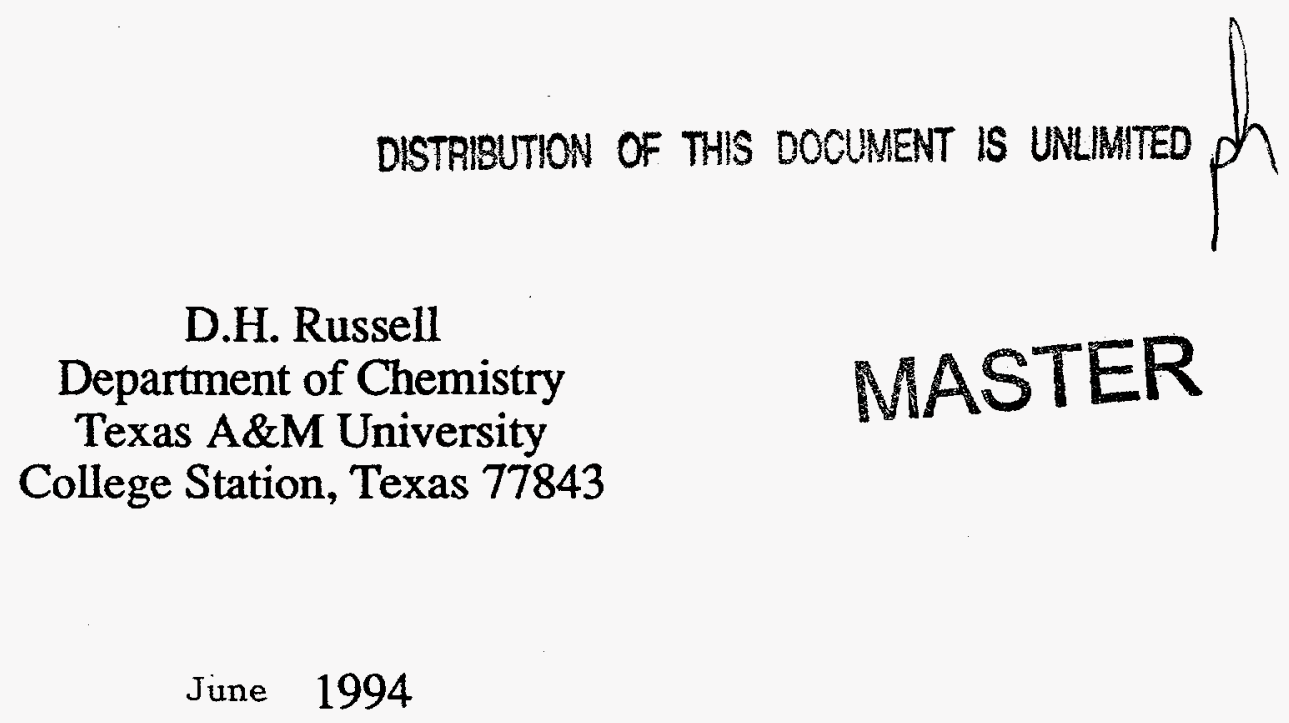

PREPARED FOR THE U.S. DEPARTMENT OF ENERGY

UNDER GRANT NUMBER DE-FG05-85ER13434 


\section{Project Summary}

This project emphasizes the development of laser mass spectrometry methods for fundamental and applied studies of gas-phase processes. The current studies are focussed on the photochemistry and photophysics of peptides and other biological molecules. Matrix-assisted laser desorption ionization (MALDI) is used to produce ions that are subsequently subjected to photoexcitation and dissociation. MALDI is still very much in the developmental stages, thus a significant portion of our research focusses on fundamental studies of the MALDI ion formation/energy transfer process. Our view is that excited state $\mathrm{H}^{+-}$-transfer reactions play an important role in MALDI, consequently a significant portion of our research activities are focussed on such studies. Fundamental studies of the role of the matrix in MALDI are an integral part of this project. A new MALDI experiment, MALDI of aerosol particles generated from solutions, has been demonstrated and new developmental research in this area is planned. We are also actively pursuing a research program on gas-phase $\mathrm{H}^{+-}$-transfer processes that mimic the MALDI process. In addition, we are developing photodissociation experiments, based on tandem time-of-flight mass spectrometers, for structural characterization of complex organic molecules. The photodissociation studies use MALDI as the ionization method. These research areas involve the development of new instrumentation, new instrument methodologies, and data processing.

\section{DISCLAIMER}

This report was prepared as an account of work sponsored by an agency of the United States Government. Neither the United States Government nor any agency thereof, nor any of their employees, makes any warranty, express or implied, or assumes any legal liability or responsibility for the accuracy, completeness, or usefulness of any information, apparatus, product, or process disclosed, or represents that its use would not infringe privately owned rights. Reference herein to any specific commercial product, process, or service by trade name, trademark, manufacturer, or otherwise does not necessarily constitute or imply its endorsement, recommendation, or favoring by the United States Government or any agency thereof. The views and opinions of authors expressed herein do not necessarily state or reflect those of the United States Government or any agency thereof. 


\section{DISCLAIMER}

Portions of this document may be illegible electronic image products. Images are produced from the best available original document. 


\section{Progress Report for the Period December 1991 through November 1994}

The research activities on this project are divided into two categories, (i) instrumentation and methods development and (ii) fundamental studies of photochemistry and photophysics of gas-phase ionic species. Obviously, there is considerable interplay between these two areas. The activities in each of these areas for the past three years are summarized below.

A. Development of New Instrumentation for Studies of Photochemistry and Photophysics A significant portion of our research effort focusses on development of new instrumentation and instrument technology for ion chemistry and analytical mass spectrometry. The initial objectives of our program centered on investigations of fundamentals of photodissociation, but over the past five years our efforts have expanded to include other aspects of photochemistry and photophysics of general importance to mass spectrometry. In particular, our research efforts on fundamentals of matrix-assisted laser desorption ionization (MALDI) have increased, especially in the area of studies of proton transfer reactions. As a direct result of more emphasis on the role of $\mathrm{H}^{+}$-transfer reaction in MALDI we have directed a significant amount of our instrument oriented research into the area of time-of-flight (TOF) mass spectrometry. Four such instruments that are frequently used in the DOE/OBES funded program are briefly described below.

\section{Electrostatic Energy Analyzer/Time-of-Flight Mass Spectrometry (E-TOF)}

The E-TOF instrument (Figure 1) is now fully operational for fundamental studies on the mechanism of ion formation in MALDI. Although kinetic energies of ions formed by desorption ionization processes can be measured by using linear-TOF instruments, many factors can lead to signal broadening in the TOF measurement. e.g., spatial and temporal ion spreads and velocity distribution. The E-TOF experiment provides a direct method for determining kinetic energies of ions, and the combined measurements of ion kinetic energy and flight time eliminates or reduces inferences.

2. Tandem Time-of-Flight Instruments (TOF/TOF) Our initial studies on tandem TOF mass spectrometry were performed using the hybrid magnetic sector/RTOF instrument. Although this instrument technology is very useful for studies of small molecules (up to $\mathrm{m} / \mathrm{z} 2000$ ), the utility for studies of large molecules and use of pulsed laser are limited. The research component of this program now focusses on instruments where MS-I and MS-II are both TOF systems. The instrument currently in use (Figure 2) is a relatively simple R-TOF design, thus MS-I and MS-II are linear TOF systems. This instrument can also be operated as three linear TOF systems, that is MS-I can be operated as a two-stage acceleration region. The initial funding for this instrument was provided by a grant from the Texas Advanced Technology Program. This instrument is now used primarily $(>70 \%)$ for DOE/OBES research projects, and the remaining $30 \%$ is dedicated to collaborative projects with 
Figure 1

\section{Energy Analysis of MALDI lons Using a Hybrid E-TOF}

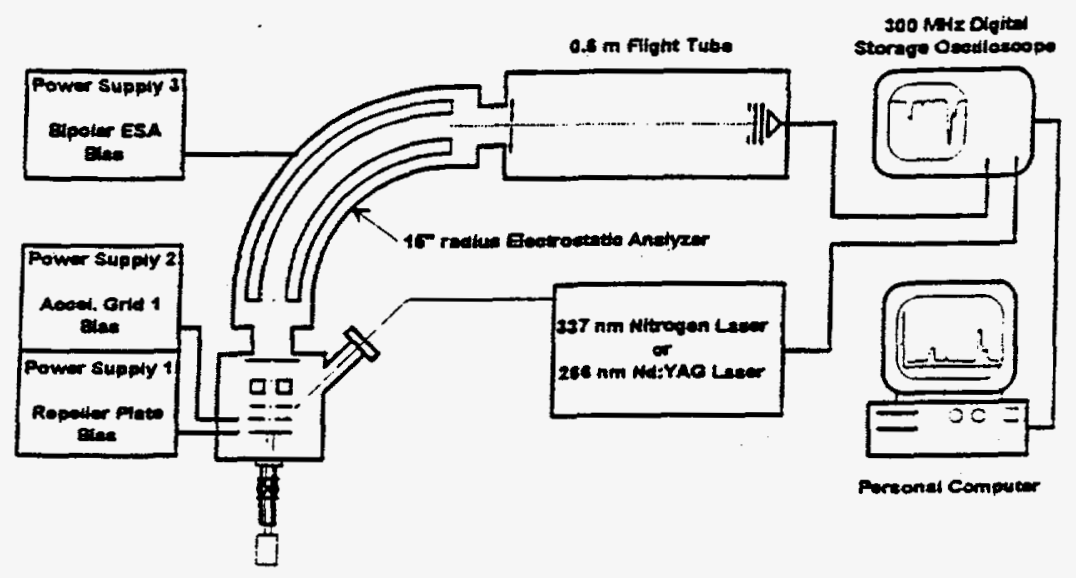

Figure 2 Schematic drawing of the tandem TOF/TOF instrument.

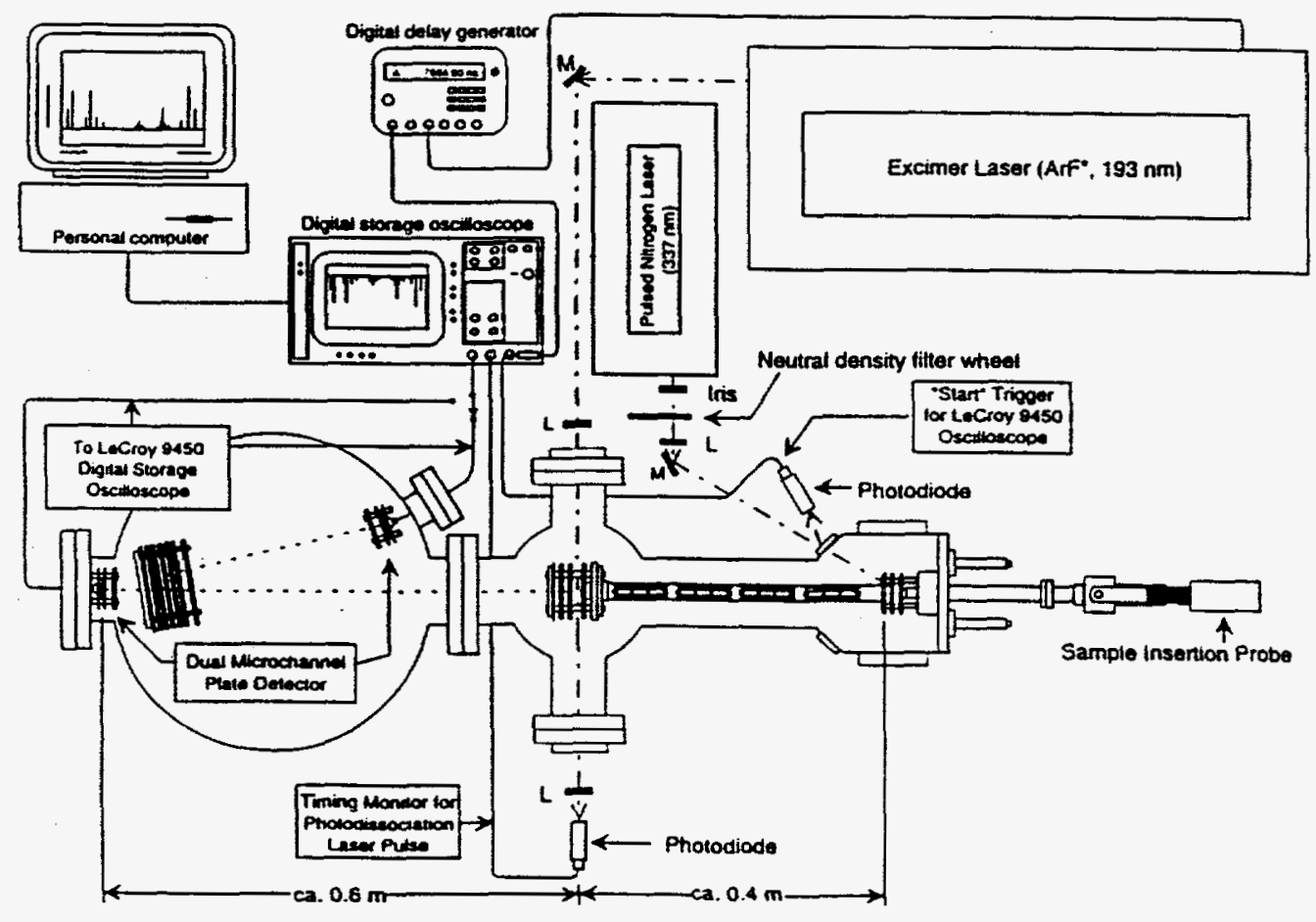


faculty or non-university research projects.

3. Jet Expansion TOF Instrument for Studies of $\mathrm{H}^{+}-$Transfer Reactions within Clusters We have just recently completed a linear TOF instrument that is fitted with high speed pumping and pulsed cluster expansion sources. (Figure 3) This system is specifically designed for studies of excited state $\mathrm{H}^{+-}$-transfer reactions of clusters. This instrument can be fitted with a reflectron to facilitate tandem mass spectrometry studies. The funding for this instrument was also provided by the Texas Advanced Technology Program. This instrument is used mostly $(-50 \%)$ for DOE/OBES research. The instrument is also being used for $\mathrm{NIH}$ related studies and by Prof. D.W. Goodman's research group (also DOE/OBES funded).

4. Hybrid Magnetic Sector/R-TOF Instruments for Measuring Arrival-Time Distributions of Mass-Selected Ions As noted above, this instrument is now routinely operated by graduate students in my group and used by other instrument users. This instrument has great utility for tandem mass spectrometry, but limits the scope of photodissociation experiments. Consequently, most of the instrument use by my group is for measurements of arrival-time distributions of mass-selected ions (see B.1. below) and more routine mass spectrometry.

\section{B. Fundamental Studies of Matrix-Assisted Laser Desorption Ionization (MALDI)}

1. Kinetic Energies of MALDI Ions A useful diagnostic probe for MALDI is the measurement of ion kinetic energies. Early in our MALDI studies we measured arrival-time distributions of MALDI generated ions and noted that (i) ion arrivaltime distributions were bimodal, a and (ii) the kinetic energies of the two ion populations differed. That is, the prompt ion population is composed of high kinetic energy ions, whereas the slow ion population is composed of low kinetic energy ions. Since making these initial observations we have focussed attention on developing additional probes to measure ion energies and temporal profiles.

a. Measurements using the E-TOF Instrument We have used this experiment to show that two types of ions are formed. A portion of the ions have a positive energy defect, e.g., kinetic energies greater than the ion source energy, but the largest fraction of the ions have a negative energy defect. Our present interpretation of these data are that the negative energy defect ions are formed "off-the-surface" from species that initially leave the surface as neutrals.

Kinetic energy distributions (Figure 4) of matrix and analyte ions have been measured for several matrices, 4-nitroaniline, 7-hydroxy-4-methylcoumarin, alphacyano-4-hydroxycinnamic acid. All measured kinetic energy distributions extend from approximately $+75 \mathrm{eV}$ to $-75 \mathrm{eV}$ at a source electric field of $10 \mathrm{kV} / \mathrm{cm}$. Two

a See Figure 2, p757, F. H. Strobel; L. M. Preston; K. S. Washburn; D. H. Russell Anal. Chem. 1992, 64, 754-762. 
Figure 3 Schematic drawing of the cluster TOF instrument.

\section{Jet-Expansion Time-of-Flight}

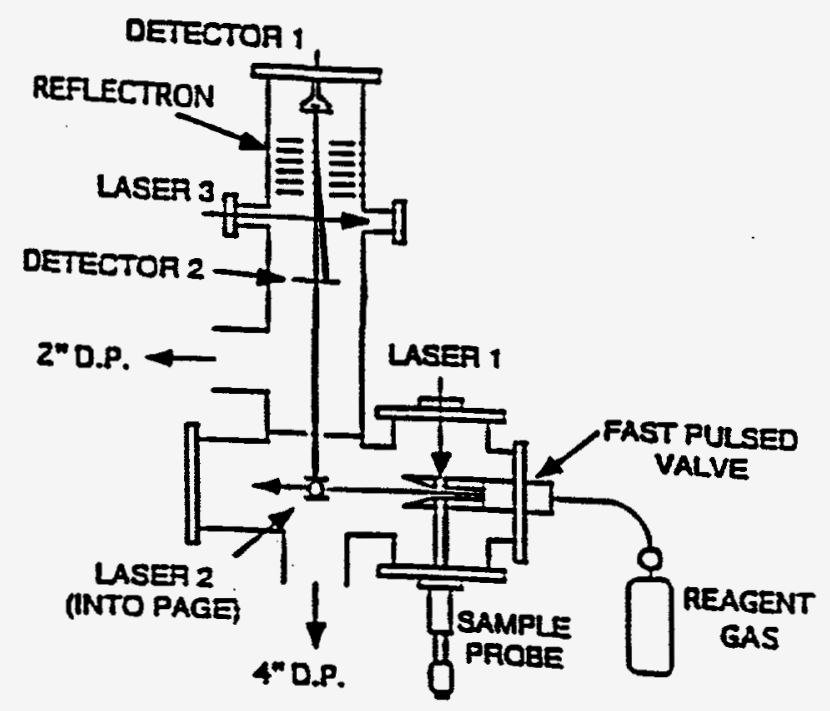

Figure 4 Plot of signal intensity versus ion kinetic energy for (a) ions used as instrument calibrants and (b) matrix (m/z 177) and analyte ions (m/z 1060).

7-OH-4-methylcoumarin / Bradykinin

$337 \mathrm{~nm}$ MALDI

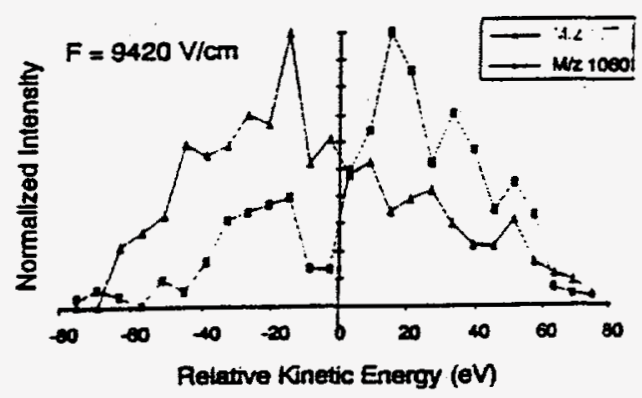

$266 \mathrm{~nm}$ Laser Desorption

of $\mathrm{m}$-Xylene

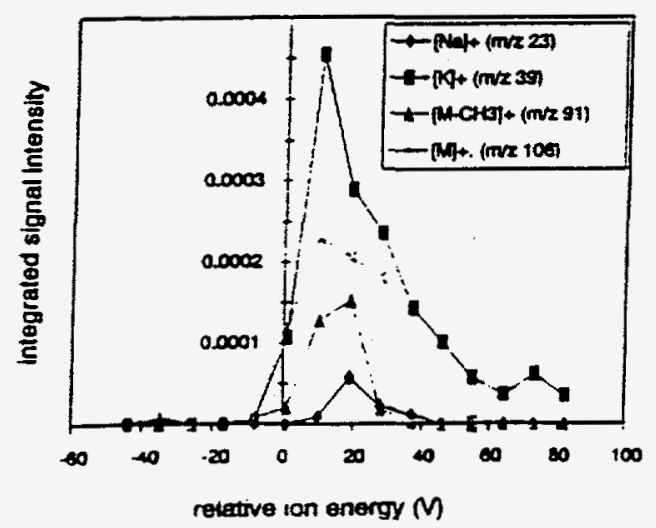


matrices (4-nitroaniline and 7-hydroxy-4-methylcoumarin) show distinct and reproducible minimum in the measured energy distribution around $0 \mathrm{eV}$. For these matrices the most probable positive energy is $c a .+15-20 \mathrm{eV}$ and the most abundant negative energy is $-20-25 \mathrm{eV}$ at $10 \mathrm{kV} / \mathrm{cm}$. These data are based on careful calibration of the energy scale of the E-TOF instrument. The energy calibration is performed by using gas-phase MPI and laser desorption of $\mathrm{Na}^{+}, \mathrm{K}+$, and chemisorbed molecules such as xylene and toluene. A paper describing the preliminary data and the calibration procedure has been submitted.

We have examined these data using several different models for the ion formation process. For ions with negative energy defects, calculations performed assuming the ions are formed at the probe surface with a potential reduced to match the ion energy deficit do not agree with the experimentally measured flight times. A calculation using the first order approximation that the ions are formed in the gas phase at a potential energy corresponding to the relative energy deficit also does not give agreement between calculated and measured flight times. If the time error between the calculated and measured flight time is associated with the time required for the neutral to reach a distance above the surface (a particular electrostatic potential) an approximate velocity for the precursor material can be estimated. The velocities we estimate for the neutral precursor is $500-600 \mathrm{~m} / \mathrm{s}$. Thus, our model, predicts that neutral species are ejected from the surface with a velocity of 500-600 $\mathrm{m} / \mathrm{s}$ and that ionization occurs approximately $50 \mu \mathrm{m}$ off the surface.

b "Design and Calibration of an Electrostatic Analyzer / Time-of-Flight Mass Spectrometer for Measurement of MALDI Ion Kinetic Energies", G. R. Kinsel, K. Gillig and D. H. Russell, J. Am. Soc. Mass Spectrom. (submitted).

"Kinetic Energies and Flight Time Analysis of MALDI Matrix Ions by Electrostatic Analyzer / Time-of-Flight Mass Spectrometry", G. R. Kinsel and D. H. Russell, (in preparation).

"Studies on the Dynamics of MALDI Ion Formation," G. R. Kinsel; K. Gillig; R. D. Edmondson; D. H. Russell, 42nd ASMS, Chicago, May 29-June 3, 1994, paper MOA1130.

b. Profile Analysis of Bovine Insulin Cluster Ion Signals We have developed a recipe for MALDI of bovine insulin (and other large peptides) that can be used to form singly charged cluster ions consisting of as many as twenty molecules. Our initial idea was to use such cluster ions for mass calibration, but we noted that the calibration curve had a y-intercept inconsistent with the performance characteristics of the instrument. The $y$-intercept is determined by the time delay between firing of the laser and triggering of the spectrum acquisition. This delay should be approximately $25 \mathrm{~ns}$ vis-a-vis the $400 \mathrm{~ns}$ obtained from the cluster calibration curve.

b In the Progress Report, papers and presentations with direct relevance to that section are listed with that section. 
We noted that the peak profiles for the cluster ions appeared to be skewed to low or high mass (depending on the exact source tuning conditions and the $\mathrm{m} / \mathrm{z}$ of the cluster) (Figure 5). We used a computer program to decompose the peaks and found the best fit was obtained using a two component profile; the best fit yields a narrow and a diffuse component. Generation of a mass calibration curve using only the narrow component gave excellent calibration curves with a y-intercept of $25 \mathrm{~ns}$.

We assume that prompt ions are formed at the surface and during the laser pulse and correspond to the narrow peak profile, whereas ions formed off the surface would correspond to the broad profile.c Although, the broad component occurs at longer flight times (relative to the narrow component) for the low mass cluster ions $(n<3)$, it occurs at shorter flight times for the higher mass cluster ions $(n>3)$. If it is assumed that the broad component is the result of delayed ionization in the gas-phase and that the neutral precursor of the ions contributing to the broad component leave the surface with some initial velocity, then the velocity of the broad component should be mass dependent. That is, the fraction of the total kinetic energy of the ion due to the initial velocity increases with increasing $\mathrm{m} / \mathrm{z}$. The "crossover point" will depend on the source region field strength, the point of gas-phase ionization, and the velocity of the precursor. Modeling of the ion flight times was performed to determine the point of ion formation and precursor velocity; best fit was obtained for velocities of approximately $900 \mathrm{~m} / \mathrm{s}$ and ionization at about 35 microns from the surface.

Two-stage acceleration measurements support the above mechanism. For instance, the flight time of the broad component can be observed to shift relative to the sharp component as a function of the source region electric field. In addition, there are indications that the actual mechanism(s) of ion formation change as a function of field strength. The broad component becomes the dominant process at low extraction field and the sharp component is dominant at high extraction fields. We are presently examining the dependence of the broad and sharp components on radial kinetic energies of the ions, i.e., low extraction field measurements would discriminate against ions having large radial kinetic energies.

"MALDI Ion Formation Dynamics Probed by Calibration of High Molecular Weight Protein Clusters", R. D. Edmondson, G. R. Kinsel and D. H. Russell, (in preparation).

"Line Width Analysis of MALDI Ion Signals: Indications of Two Ion Formation Processes", R. D. Edmondson, G. R. Kinsel and D. H. Russell, (in preparation).

c We have considered many arguments concerning the two component peak profiles. A frequently raised question is whether the broad component is due to metastable dissociation. Cluster ions dissociate to form smaller clusters or the monomer ion not by loss of small neutral molecules, thus dissociation would give rise to tailing on the high mass side of the peak. 
Figure 5 Peak profiles and decomposed peak profiles for bovine insulin cluster ions.

Two Component Fits of Bovine Insulin Cluster lons
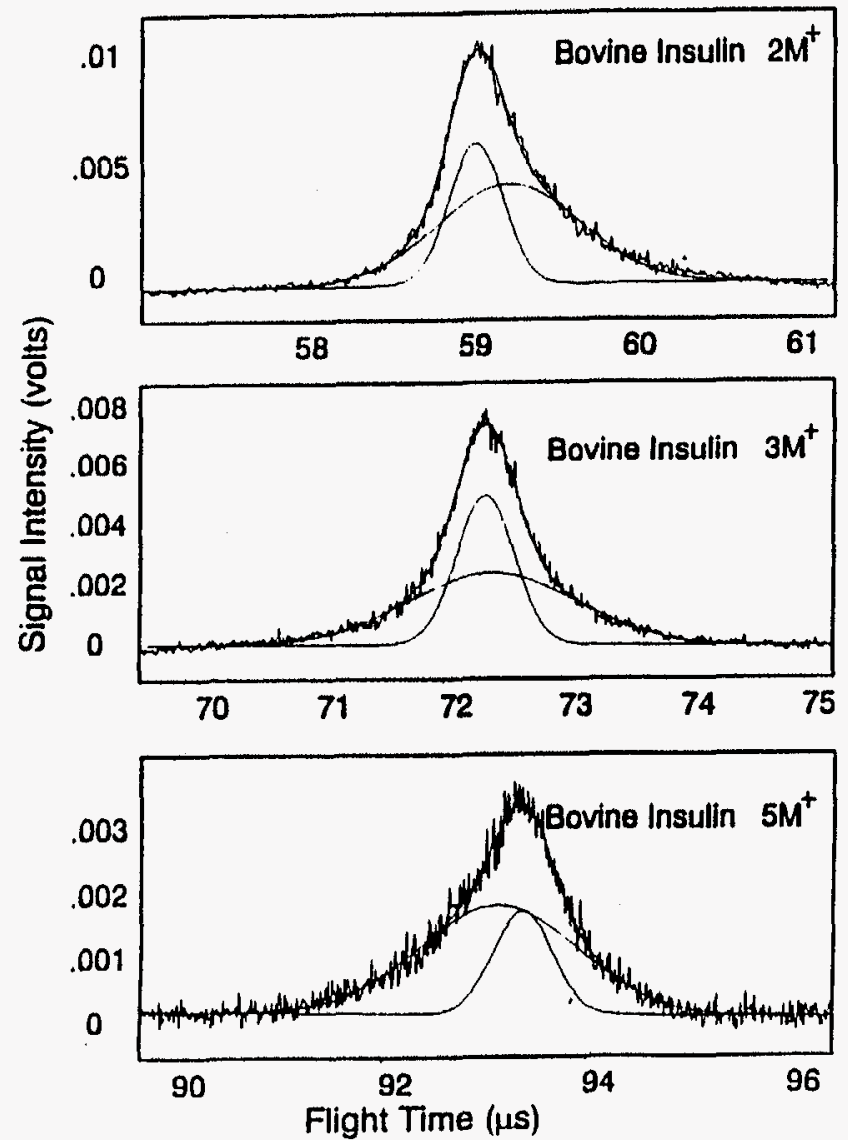

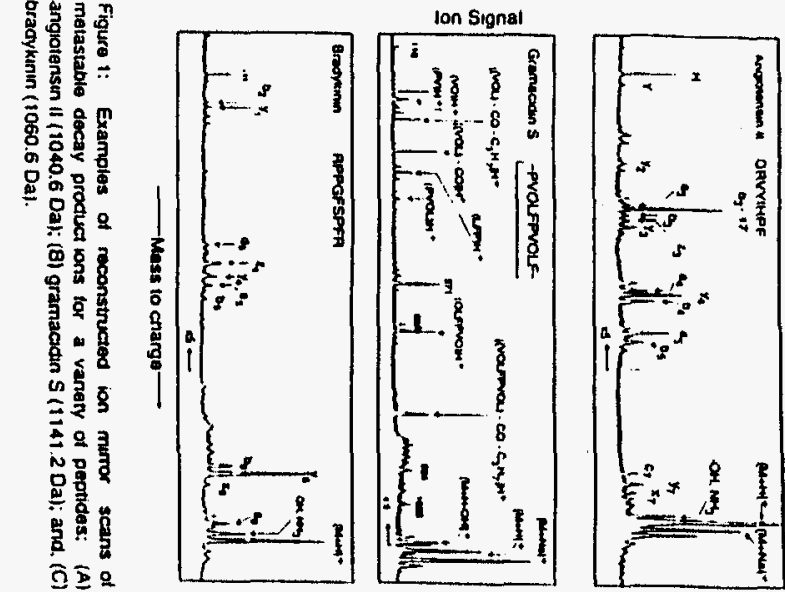

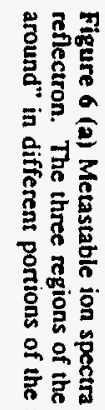

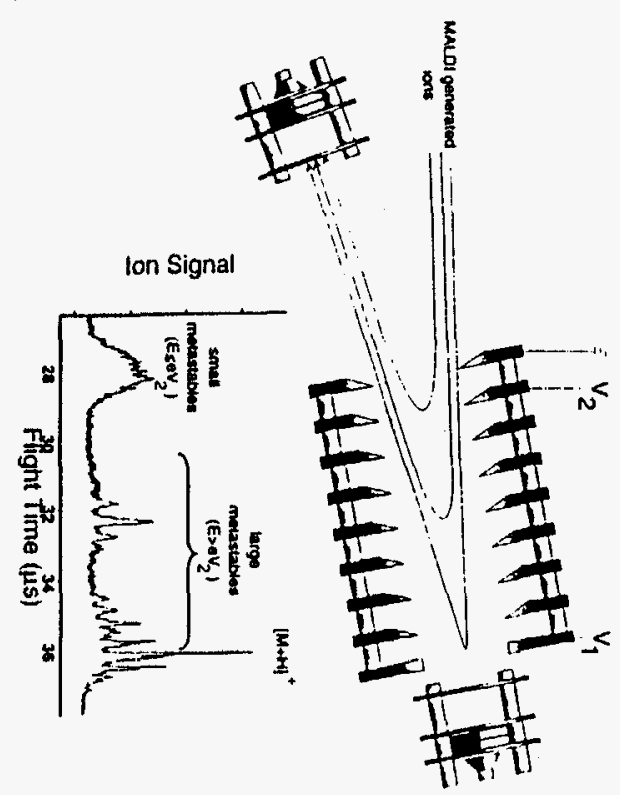


Formation Processes", R. D. Edmondson, G. R. Kinsel and D. H. Russell, (in preparation).

2. Metastable Ion Dissociation Studies MALDI metastable product ion spectra have been acquired for bradykinin, angiotensin I, angiotensin II, mellitin, alpha-melanocyte, gramicidin $S$ and bovine insulin using a scanned ion mirror technique in a R-TOF MS (Figure 6a). Application of this method involves stepping the voltages applied to the deceleration grid and reflector grid of a two stage reflectron TOFMS across a range of voltages from ground to greater than the acceleration voltage (Figure 6.b.). All fragment ions with energies $\left(E_{f}=E_{p} * m_{F} / m_{P}\right)$ less than the potential applied to the deceleration grid are reflected in the first stage of the two-stage reflectron and detected as a broad unresolved bump in the mass spectrum. Fragment ions with energies greater than the potential applied to the reflector grid are transmitted through the reflector. Fragment ions with energies between the deceleration potential and the reflector potential are separated and reflected in the second stage of the two stage R-TOFMS. These latter ions are detected as well resolved ion signals in the mass spectrum. Mass assignment of the ion signals is performed by using a procedure we have developed. The masses of the product ions are determined by fitting calculated and experimental flight times using known conditions and a SIMPLEX regression routine.

We are particularly interested in the source of the internal energy that leads to subsequent metastable decay of the analyte ion. The extent of metastable ion decay was quantified by first measuring the total MALDI signal (ions and neutrals) and then measuring the yield of neutral metastable products. This measurement is made by placing a strong retarding field at the end of the flight tube thereby allowing only the neutral material to be transmitted. The influences of desorbing laser intensity, matrix-to-analyte ratio and matrix choice have been investigated thus far. The results can be summarized as follows: 1) the effect of laser intensity on metastable decay appears to be analyte and possibly matrix dependent - for matrix/non-absorbing analyte combinations no effect of increasing laser intensity is observed, for other matrix/absorbing analyte combinations metastable decay increases with increases in desorbing laser intensity, 2) changes in M:A ratio, at least above ca. 250:1, appear to have little effect on total metastable decay, 3) matrix choice seems to have an influence on metastable decay but the effect is dependent on the nature of the analyte, i.e. peptides and proteins show significantly less variation in metastable decay as a function of matrix than does vitamin $B_{12}$.

"Factors Affecting Metastable Decay Rates of MALDI Generated Ions", M. E. Gimon, G. R. Kinsel and D. H. Russell, (in preparation).

"Metastable Fragmentation of Absorbing and Non-absorbing Analytes during 337 nm Matrix Assisted Laser Desorption Ionization", M. E. Gimon, G. R. Kinsel and D. H. Russell, (in preparation). 
3. Role of Matrices in Ion Formation Studies in this area focus on questions concerning the mechanism of ion formation by MALDI and issues regarding the use of MALDI for producing ions that are probed by gas-phase photodissociation experiments. Highlights of this work are: (i) Both prompt and metastable decay is significantly reduced for ions desorbed from nitrocellulose over ions desorbed directly from metal surfaces, (ii)) Metastable decay rates have been calculated for several analytes by measuring the numbers of neutral product ions produced at several different points along the drift region. Rates of $10^{3}$ to $10^{4} \mathrm{sec}^{-1}$ are obtained, (iii) studies comparing the identities of the metastable fragment ions with prompt fragment ions recorded in linear TOFMS mass spectra and with fragment ions observed by tandem mass spectrometry or FT-ICR are underway. More extensive fragmentation is observed in the TOF metastable spectra than in the ICR spectra. We attribute such effects to ion collection efficiencies but additional studies are needed, (iv) conditions have not been identified which totally eliminate metastable decay of the MALDI analyte ions on the TOFMS time scale. Although the absolute yield of fragment ions is small at low desorbing laser intensities, the most important parameter appears to be the matrix. That is, different amounts of fragmentation is observed with different matrices; alpha-hydroxycinnamic acid produces the most fragmentation, and the heavy-atom ( $\mathrm{Br}$ or I) substituted matrix compounds appear to yield the lowest abundance of fragment ions.

We have performed a number of studies that are best classified as probing the role of matrices in the MALDI process. The working hypothesis (for positive ion MALDI) is that formation of the analyte $[\mathrm{M}+\mathrm{H}]+$ ion involves an acid/base reaction between the basic analyte and acidic matrix. The $\mathrm{H}^{+-}$transfer is initiated by photon absorption by the matrix, i.e., a photon-induced "pH-jump" experiment. Thus, processes that alter the relaxation dynamics of the photoexcited acidic molecule, e.g., increase or decrease the probability of internal conversion, promote intersystem crossing, or lead to intermolecular energy transfer, directly influence the analyte ion yield. Similarly, substitution of functional groups attached to the matrix molecule that alter the acidity of the molecule also alter the analyte ion yield.

"Are Proton Transfer Reactions of Excited States Involved in UV Laser Desorption Ionization?", M. E. Gimon, L. M. Preston, T. Solouki, M. A. White and D. H. Russell, Org. Mass Spectrom., 1992, 27, 827-830.

"The Role of the Matrix in Matrix-Assisted Laser Desorption Ionization: What We Know Versus What We Understand," D. H. Russell 42nd ASMS, Chicago, May 29-June 3, 1994, paper MOA0930

i) Heavy atom effect If the lowest-lying singlet excited state of the matrix compound is directly involved in the MALDI excited state $\mathrm{H}^{+-t r a n s f e r ~ r e a c t i o n, ~ t h e n ~}$ substitution of a heavy atom onto the ring should alter the abundance of the $[\mathrm{M}+\mathrm{H}]+$ ion of the analyte in the MALDI mass spectrum. Pumping of the $S_{2} \leftarrow S_{0}$ rather than 
the $S_{1} \leftarrow S_{0}$ band also shows marked differences in the MALDI mass spectra and the yield of analyte ions (Figure 7). This result illustrates two general arguments: (i) MALDI involves more than simply deposition of energy into the solid sample, e.g., it matters which electronically excited state of the matrix is populated. (ii) Because the decay dynamics of $S_{2}$ and $S_{1}$ and the MALDI mass spectra obtained at 266 and 337 $\mathrm{nm}$ are different, suggest that the MALDI effect is a direct result of the electronically excited species vis-a-vis simply due to a rovibronically excited species. If the $T_{1}$ state formed by intersystem crossing is more acidic than the $S_{1}$ state, heavy atom substitution may increase the $[\mathrm{M}+\mathrm{H}]+$ ion yield. Conversely, if the $\mathrm{T}_{1}$ state is less acidic, then the yield of the $[\mathrm{M}+\mathrm{H}]+$ ion should decrease. We have performed such studies on a number of heavy atom and non-heavy atom substituted matrix compounds. Two general observations were made: (i) the ion yield is greater for the heavy atom substituted compound, and (ii) the abundance of analyte fragment ions is decreased for the heavy atom substituted matrices. Therefore, we conclude that: 1) matrix excited states are involved in the $\mathrm{H}^{+}$-transfer process, 2) these studies argue against involvement of radical cation species because heavy atom substituted benzenes are inefficiently photoionized by 2 photon absorption, especially $\mathrm{Br}$ and I substituted compounds, 1 and 3 ) triplet states and/or longer lived excited states may be more amenable for proton transfer to analyte species.

"Effects of Heavy Atom Substituents on Matrices used for Matrix-Assisted Laser Desorption Ionization Mass Spectrometry", L. M. Preston-Schaffter, G. R. Kinsel and D. H. Russell, J. Am. Soc. Mass Spectrom., (in press).

ii) Vitamin B12 This molecule was used to probe for MALDI because it ionizes by direct (without matrix) laser desorption ionization to yield fragment ions and by MALDI to give $[\mathrm{M}+\mathrm{H}]+($ some $[\mathrm{M}+\mathrm{Na}]+)$ and $[\mathrm{M}+\mathrm{H}-\mathrm{CN}]+$. From these studies we conclude: (i) the amount of energy transferred to the analyte is primarily dependent on the matrix, (ii) analyte fragmentation is not dependent on M:A ratio above a threshold value nor does direct photodissociation of the analyte play aa significant role in the fragmentation process, (iii)) fragmentation increases with increasing laser intensity for alpha-cyano-4-hydroxycinnamic acid but not for the heavy atom substituted matrix, (iv)) the results support the idea that the exothermicity of the MALDI proton transfer reaction determines the internal energy and subsequent fragmentation of the analyte molecule, thus, analyte activation is matrix dependent, (v) perhaps the key conclusion of this study is that not only must the matrix be acidic enough to transfer a proton to the analyte, but also the analyte must be sufficiently rugged to withstand the energy associated with the proton transfer reaction i.e. the energetics (and efficiency) of the matrix to analyte proton transfer reaction must be viewed as a window - with the lower bound set by the requirement that the proton transfer reaction be exothermic and the upper bound set by the lability of the molecule. 
"Fragmentation of Vitamin B12 during $337 \mathrm{~nm}$ Matrix Assisted Laser Desorption / Ionization", G. R. Kinsel, L. M. Preston and D. H. Russell, Biological Mass Spectrom., 1994, 23 , 205-211..

"Fragmentation from Peptides using Matrix-Assisted Laser Desorption Ionization with Time-of-Flight Mass Spectrometry", L. M. Preston-Schaffter, G. R. Kinsel and D. H. Russell, (in press).

iii) Taxol and taxanes Analytes other than peptides and proteins can be analyzed by MALDI but these compounds are more sensitive to the matrix used. For example, "conventional" MALDI matrices failed to produce intact ions of taxol and taxanes but 4-nitrophenol produced abundant $[\mathrm{M}+\mathrm{H}]+$ ion signal. Fragment ions are always observed in the taxol MALDI mass spectra and the relative abundances are dependent on both the matrix and the laser irradiance. The results are interpreted as suggesting that taxol is both fairly acidic and fairly labile and as such requires an acidic matrix. Our current focus is to use similar logic to develop MALDI recipes for relatively non-polar polymers and other non-peptide molecules, esp. DNA.

"Matrix-Assisted Laser Desorption / Ionization Time-Of-Flight Mass Spectrometry of Taxol and various Taxanes", M. E. Gimon, G. R. Kinsel, R. D. Edmondson, D. H. Russell, T. R. Prout and N. Ewald, J. Nat. Prod., (in press).

iv) Studies of Nitroaniline and Coumarin compounds as matrices Studies that compare the MALDI effect for structurally different molecules provide useful data for analytical utility but such studies can also reveal fundamental understanding about the ionization process. Sinapic acid (3,5-dimethoxy-4hydroxycinnamic acid), a standard MALDI matrix, is an acidic matrix due to the 4position $\mathrm{OH}$ group. For example, the methyl ester of sinapic acid is at least as good, possibly better, a matrix as the free acid. Likewise, nitroanilines and coumarins are effective matrices. The fact that 4-nitroaniline and 2,4-dinitroaniline are effective proton donor (acidic) matrices provides the basis for our hypothesis on excited state processes in MALDI. Furthermore, the utility of compounds that have no labile protons coumarins as MALDI matrices suggests that photochemistry plays a role in altering the form of the matrix prior to the MALDI ionization process. The correlation between Hammett para sigma minus values and MALDI $[\mathrm{M}+\mathrm{H}]+$ analyte ion yields for a series of 4 substituted aniline matrices, and the correlation between MOPAC calculated $\triangle \mathrm{pKa}$ for the excited state of the substituted anilinium ion for the same series of 4 substituted aniline matrices and MALDI analyte ion yields collectively present a strong case for the involvement matrix excited state species in the MALDI ionization process. In addition, these data argue against the involvement of a photoionized (radical cation or self protonated) matrix ions in the MALDI process. Several papers on these subjects are listed below; however, because 
much of this is still an active research area and further discussion is presented in the proposal text.

"Coumarin Matrices Used in Matrix-Assisted Laser Desorption Ionization Mass Spectrometry", L. M. Preston-Schaffter and D. H. Russell, Anal. Chem. (submitted).

"Effects of Matrix Structure and Acidity on Ion Formation in Matrix Assisted Laser Desorption / Ionization Mass Spectrometry", L. M. Preston-Schaffter, M. E. Gimon, G. R. Kinsel and D. H. Russell, J. Am. Soc. Mass Spectrom. (submitted).

"The Role of Matrix Acidity in MALDI Ion Formation", M. E. Gimon, L. M. Preston-Schaffter and D. H. Russell, Presented at the 42nd ASMS Conference on Mass Spectrometry and Allied Topics, Chicago, IL, June 1994, paper Th50.

v) Studies of MALDI threshold irradiance We have performed studies on the laser irradiance threshold for MALDI. The results can be summarized as follows: (i) We do not observe a direct correlation between the absorbance of the matrix and the threshold irradiance for analyte ion production, (ii) a better correlation is found between threshold irradiance and matrix luminescence, (iii) matrix absorption is inversely correlated with the optimum $\mathrm{M}: \mathrm{A}$ ratio for abundant $[\mathrm{M}+\mathrm{H}]+$ yield i.e. strongly absorbing matrices show favor lower M:A ratios than weakly absorbing matrices, (iv) the results suggest that some form of total energy balance must be considered when defining experimental conditions for a given matrix, i.e., both the energy absorbed and luminescence of the solid should be considered as parameters that influence the MALDI effect, (v) luminescence studies could provide essential information for understanding MALDI ion yields and energy transfer.

"Comparison of Laser Energy Thresholds for Matrix Assisted Laser Desorption Ion Production with Various Matrices", L. M. Preston-Schaffter and D. H. Russell, (in preparation).

vii) Nitrocellulose studies 1) nitrocellulose added to the sample preparation improves the reproducibility of MALDI analyte ion signal yields, both from spot to spot on the probe surface and from sample to sample, 2) small enhancements in both total analyte $[\mathrm{M}+\mathrm{H}]+$ ion yield and resolution are also observed, 3) Microscopy shows that crystallite size and morphology are different for samples deposited on nitrocellulose compared to those deposited on bare stainless steel, 4) improvement in ion yield and signal resolution may be a result of an enhancement of a field ionization type mechanism, i.e., the growth of small micro-needles from nitrocellulose has been used in FD-FI experiments to enhance ion production.

"Reproducibility and Quantitation of Matrix Assisted Laser Desorption Ionization Mass Spectrometry: Effects of Nitrocellulose on Peptide Ion Yields," L. M. 
Figure 7 MALDI mass spectra of bovine insulin acquired at 266 and $337 \mathrm{~nm}$. Note the difference in ion yield in both regions of the spectrum.

\section{Wavelength Dependent MALDI $\alpha$-cyano-4-hydroxycinnamic acid/ Bovine Insulin}

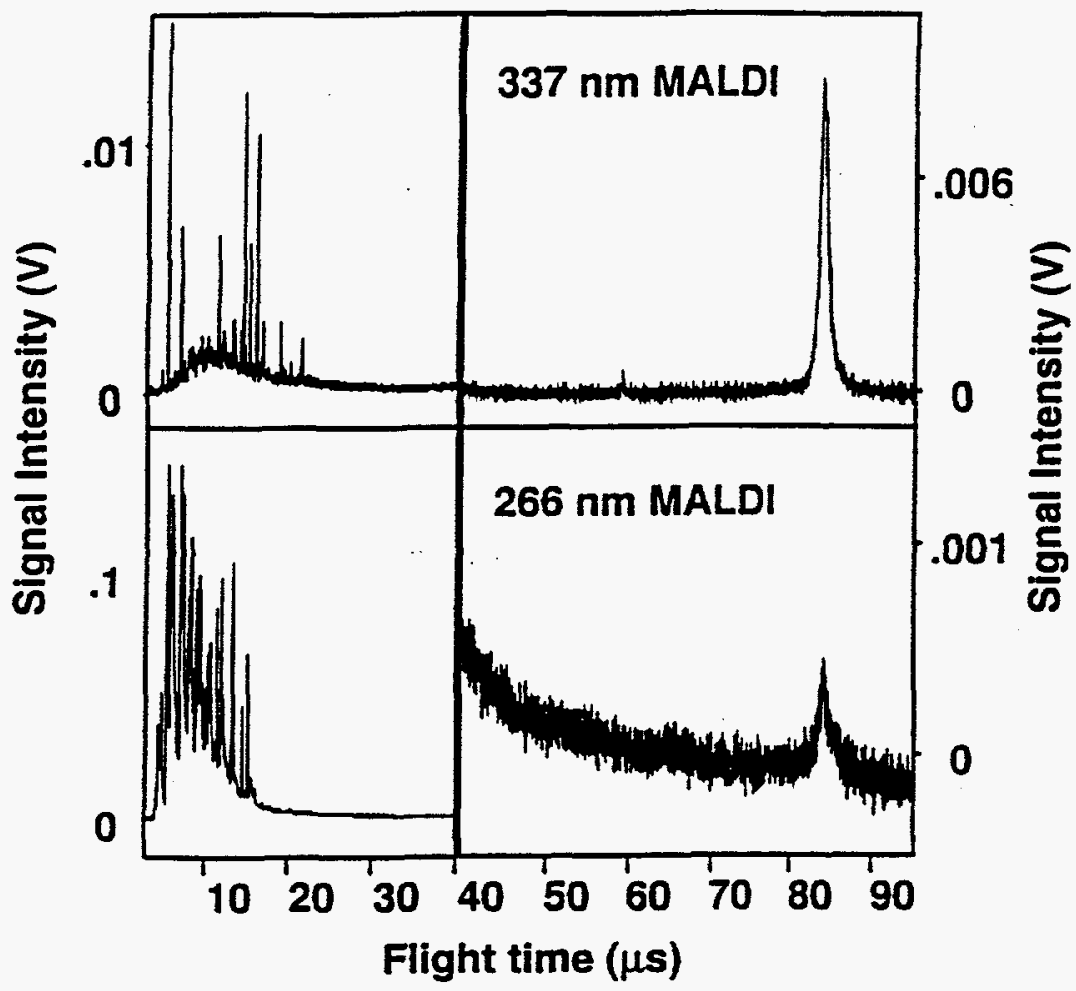

Figure 8 Photofragment ion spectrum of bradykinin. Positive going signal the photofragment ion and the negative going signal corresponds to an attentuation that ion when the laser is "on," e..g., a photodepletion signal.

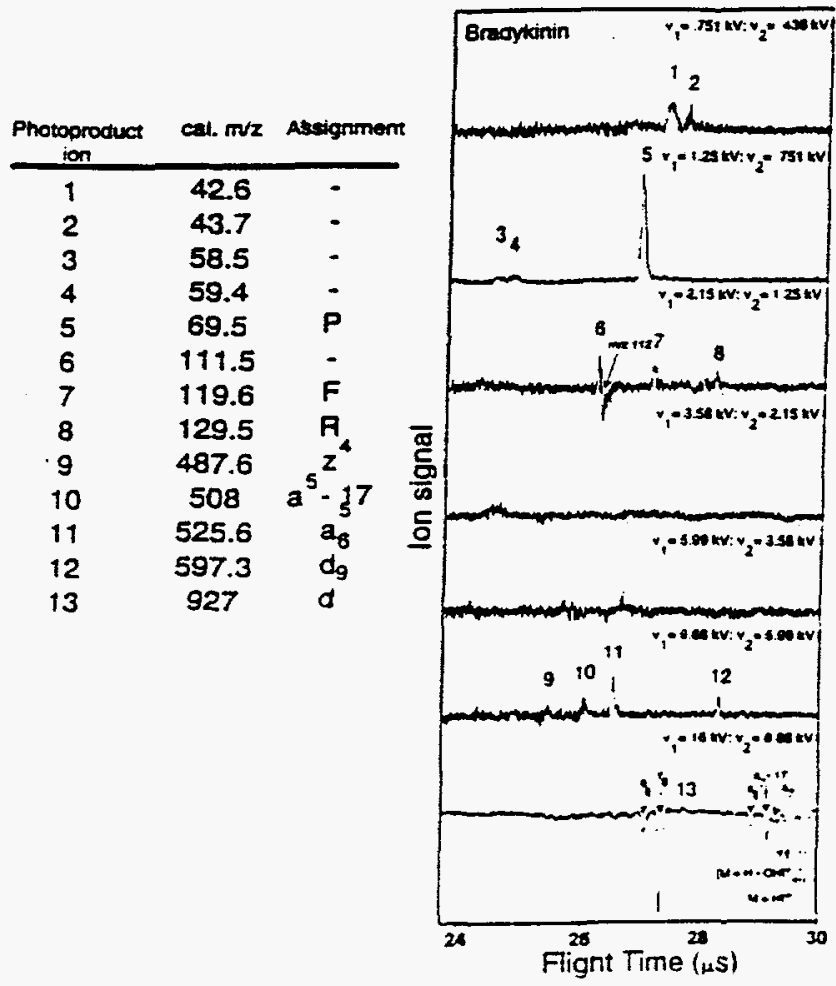


Preston, K. K. Murray and D. H. Russell, Biol. Mass Spectrom. 22, 544-550, 1993.

C. Photodissociation Studies of Ions Formed by MALDI Substantial progress has been made on the photodissociation experiment. Much of this progress has been in the area of instrumentation and development of experimental procedures. For example, the reflectron-TOF instrument is opertional and we routinely acquire metastable ion spectra (see Figure 6a) for a variety of compound classes. In addition, we have acquired preliminary photofragment ion spectra (see Figure 8) on model peptides, bradykinin and angiotensin III. The instrument can also be used for MPI of neutral species formed by metastable dissociation. This work is discussed further in the proposal. Preliminary work on these topics were presented at ASMS 1994.

"Photodissociation of High Molecular Weight Proteins in a Two-Stage Linear Time-of-Flight Mass Spectrometer", G. R. Kinsel, M. E. Gimon and D. H. Russell, (in preparation).

"Wavelength Dependent Peptide Photodissociation in a Reflectron Time-of-Flight Mass Spectrometer", M. E. Gimon, G. R. Kinsel and D. H. Russell, (in preparation).

\section{Other Publications}

"Photodissociation of Gas Phase Ions: A Review with Emphasis on Photodissociation and Structural Mass Spectrometry", M. E. Gimon and D. H. Russell, Chemical Society Specialists Report: Mass Spectrometry: Vol. 11, Ed. K. R. Jennings (in press).

"Biodegradation of Hypertrehalofemic Hormone in Blaberus discoidalis Cockroaches monitored by MALDI-MS", A. Strey, G. R. Kinsel, D. H. Russell, M. Wright and T. Hayes, (in preparation).

"Synthesis, Purification and Characterization of Manduca sexta Eclosion Hormone", Y. Wang, L. Yurttas, B. E. Dale, D. H. Russell, G. R. Kinsel, L. M. Preston, M. S. Wright and T. K. Hayes, (in preparation).

\section{CONFERENCE PRESENTATIONS}

During this report period 38 conference presentation were made. These are not listed because of space limitations.

\section{Aerosol MALDI}

1. General Studies of Aerosol-MALDI We have developed a technique for liquid introduction into a mass spectrometer that combines aerosol liquid introduction with matrix-assisted laser desorption ionization (MALDI). [Paper \#2] This laser spray ionization technique has been used to ionize a variety of peptides 
and proteins with molecular weights up to 80,000 . Laser spray ionization using aerosol MALDI promises to be a powerful method in biological mass spectrometry for coupling liquid separation techniques such as liquid chromatography (LC) and capillary electrophoresis (CE) to a mass spectrometer and mass spectrometric analysis of biomolecule solution chemistry. We are also developing a protein sequencing method that uses LC/MALDI-MS in conjunction with modified manual Edman chemistry.

In the aerosol MALDI experiment, the analyte biomolecule is combined with a UV absorbing matrix molecule in a methanol solution and flowed into a nebulizer at a total rate of 0.5 to $1 \mathrm{~mL} / \mathrm{min}$. The solution is sprayed into vacuum and the aerosol particles are desolvated as they pass through a $25 \mathrm{~cm}$ long $4 \mathrm{~mm}$ I.D. heated drying tube. Ions are formed when the collimated aerosol beam is irradiated by the $355 \mathrm{~nm}$ output of a frequency tripled Nd:YAG laser operating at $10 \mathrm{~Hz}$. The ions are accelerated to between 5 and $8 \mathrm{kV}$, mass separated in a $1.1 \mathrm{~m}$ flight tube, and detected with a microchannel plate particle multiplier. Mass spectra are averaged by a digital oscilloscope and downloaded to a microcomputer for mass calibration and analysis.

For LC/MS operation, the matrix solution is combined with the column effluent in a mixing tee. Averaged aerosol MALDI mass spectra are downloaded and stored on the microcomputer every $10 \mathrm{~s}$. In a recent paper we demonstrate LC/MALDI-MS for the reversed-phase HPLC separation of bradykinin, gramicidin S, and myoglobin. We are also performing detailed studies of the effects on aerosol MALDI ion of important HPLC parameters such as matrix and analyte concentration, matrix to analyte ratio, solution $\mathrm{pH}$, solvents, and buffers. A paper on the effects of matrix and analyte concentration and matrix to analyte ratio is near completion.

We have been evaluating two nebulizer configurations: pneumatic and combination pneumatic/ultrasonic. The pneumatic nebulizer uses two concentric stainless steel tubes, with the central tube for liquid and the outer tube for the carrier gas. The ultrasonic nebulizer uses a small capillary tube to deliver the liquid through the central bore of the nebulizer to the nebulizer tip. Carrier gas can be flowed through the central bore of the nebulizer, or in an annular configuration flowing past the nebulizer tip. The initial aerosol MALDI studies were performed with the pneumatic nebulizer and we have recently been developing the ultrasonic nebulizer. Preliminary studies with the ultrasonic nebulizer show at least a tenfold improvement in signal and an improvement in signal to noise to the point where a single laser shot has yielded an aerosol MALDI signal for gramicidin $S$ with a signal to noise of approximately 10 .

2. Studies of Laser-Induced $\mathrm{H}^{+}$-Transfer Reactions The laser ablation supersonic expansion time-of-flight apparatus for studying proton transfer in clusters is operational. The cluster source is similar to that described by Haufler. We are now working on development of methods for producing the desired cluster species (see proposal text). 


\section{PUBLICATIONS}

2. "Liquid Sample Introduction for Matrix-Assisted Laser Desorption Ionization," K. K. Murray and D. H. Russell, Anal. Chem. 65, 2534-2537, 1993.

3. "Aerosol Matrix-Assisted Laser Desorption Ionization Mass Spectrometry,"K. K. Murray and D. H. Russell, J. Am. Soc. Mass Spectrom. 5, 1-9, 1994.

4."Matrix-Assisted Laser Desorption Ionization of Aerosols: The Ionization Mechanism," K. K. Murray and D. H. Russell, In Laser Ablation: Mechanisms and Applications - II, J. C. Miller and D. B. Geohegan, Eds.; AIP Conference Proceedings 288, American Institute of Physics: New York, NY, 1994; pp. 459-464.

5."Aerosol Matrix-Assisted Laser Desorption Ionization of Aerosols for Liquid Chromatography Time-of-Flight Mass Spectrometry," K. K. Murray, T. M. Lewis, M. D. Beeson and D. H. Russell, Anal. Chem. 1994, 661601-1609.

6. "Laser Spray Ionization for Biological Mass Spectrometry," K. K. Murray and D. H. Russell, Am.Lab, (in press).

7."Aerosol Matrix-Assisted Laser Desorption Ionization: The Effects of Analyte Concentration and Matrix to Analyte Ratio," M. D. Beeson, K. K. Murray, D. H. Russell, Anal. Chem. (submitted).

8."Aerosol Vacuum Sample Deposit for Matrix-Assisted Laser Desorption Ionization," K. K. Murray and D. H. Russell, Anal. Chem.. (submitted).

9. "Aerosol Matrix-Assisted Laser Desorption Ionization: The Effects of HPLC Solvents and Buffers," M. D. Beeson, K. K. Murray, D. H. Russell, Anal. Chem. (submitted).

10."Laser Induced Metal Ejection from Aerosol Borne Metallopeptides and Metalloproteins," K. K. Murray, M. D. Beeson and D. H. Russell, (in preparation). 11."Perormance of an Ultrasonic Nebulizer for Laser Spray Ionization," K. K. Murray, X. Wei, and D. H. Russell, (in preparation).

\section{CONFERENCE PRESENTATIONS}

During this report period 22 presentations were made on the aerosol research. 